Case Report

\title{
Osteomyelitis of the Patella in a 10-Year-Old Girl: A Case Report and Review of the Literature
}

\author{
Matthias Sperl, ${ }^{1}$ Michael Novak, ${ }^{1}$ Daniela Sperl, ${ }^{2}$ Martin Svehlik, ${ }^{1}$ \\ Georg Singer, ${ }^{3}$ and Tanja Kraus ${ }^{1}$ \\ ${ }^{1}$ Department of Pediatric Orthopedics, Medical University of Graz, Graz, Austria \\ ${ }^{2}$ Division of Pediatric Hemato-Oncology, Medical University of Graz, Graz, Austria \\ ${ }^{3}$ Department of Pediatric and Adolescent Surgery, Medical University of Graz, Graz, Austria \\ Correspondence should be addressed to Matthias Sperl; matthias.sperl@klinikum-graz.at
}

Received 15 March 2017; Accepted 9 April 2017; Published 18 April 2017

Academic Editor: Johannes Mayr

Copyright (C) 2017 Matthias Sperl et al. This is an open access article distributed under the Creative Commons Attribution License, which permits unrestricted use, distribution, and reproduction in any medium, provided the original work is properly cited.

\begin{abstract}
The incidence of osteomyelitis constantly declines. While the disease most commonly affects the long bones, involvement of the patella is rarely seen. Due to this rarity and the variable clinical presentation, diagnosis is often delayed. The present case report describes a 10-year-old female patient with a delayed diagnosis of patella osteomyelitis. The diagnostic procedures and the treatment regimen are described. Additionally, a detailed literature review of the available publications reporting osteomyelitis of the patella in children is presented.
\end{abstract}

\section{Introduction}

In western industrialized countries, acute osteomyelitis represents a rare disease in children. Nevertheless, its severe sequelae such as growth disturbances, early joint destruction, and the resulting lifelong disability are well known and feared. While acute osteomyelitis of the long bones is more common, involvement of the patella is seldom encountered $[1,2]$. In 1829, Thirion described the first case of acute patella osteomyelitis [3]. Due to its variable clinical presentation, diagnosis is frequently delayed.

We report the case of an otherwise healthy 10 -year-old girl with osteomyelitis of the left patella. The patient's history and the management of the case are presented. Additionally, a review of the currently available literature reporting osteomyelitis of the patella is given.

\section{Case Report}

A 10-year-old girl complained about pain of the left knee after an accident sustained during trampoline jumping. The patient had a superficial skin abrasion but due to decreasing pain no doctor was consulted. Three weeks later she walked
35 kilometres in three days during a school trip causing moderate pain of her left knee mainly during night-time. However, the pain was not associated with swelling, redness, or fever. The family consulted a medical practitioner, a pediatrician, and an orthopaedic surgeon. Radiographs of the left knee were performed and no alterations of the patella were described. The patient was treated with NSAID.

Due to persisting pain the local hospital was consulted eight weeks after the first symptoms. Physical examination did not reveal any abnormalities except a painful leg extension. The erythrocyte sedimentation rate was $19 \mathrm{~mm} / \mathrm{h}$ (normal range: $1-10 \mathrm{~mm} / \mathrm{h}$ ) and the antistreptolysin-titer was $352 \mathrm{IU} / \mathrm{ml}$ (normal value: $-200 \mathrm{IU} / \mathrm{ml}$ ). For further diagnostics, another radiograph and a subsequent MRI of the left knee were performed showing an osteolytic lesion with a diameter of $1.5 \mathrm{~cm}$ of the patella with central necrosis (Figures 1 and 2). Radiologists and an orthopaedic surgeon interpreted the lesion as a chondroblastoma of the patella and a follow-up with MRI was planned within 3 months.

Five days later the girl returned because of increasing pain of her left knee. Clinical inspection showed swelling and hyperthermia of the left knee. The white blood cell count was $8.750 / \mu \mathrm{l}$ (normal values $5.50-13.50 / \mu \mathrm{l}$ ), the erythrocyte 

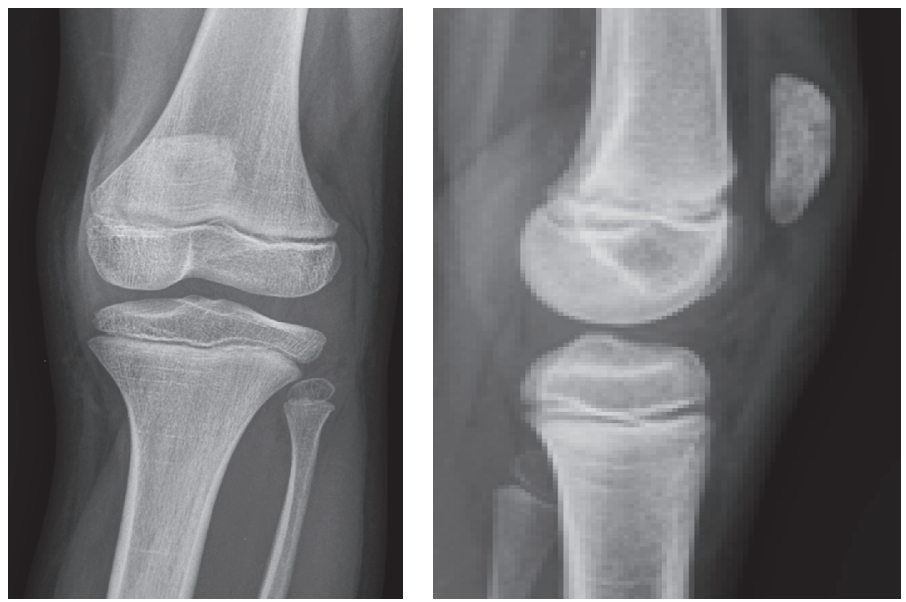

FIGURE 1: Radiographs of the knee joint 8 weeks after the first symptoms. A hypodense area is visible at the upper pole of the patella.

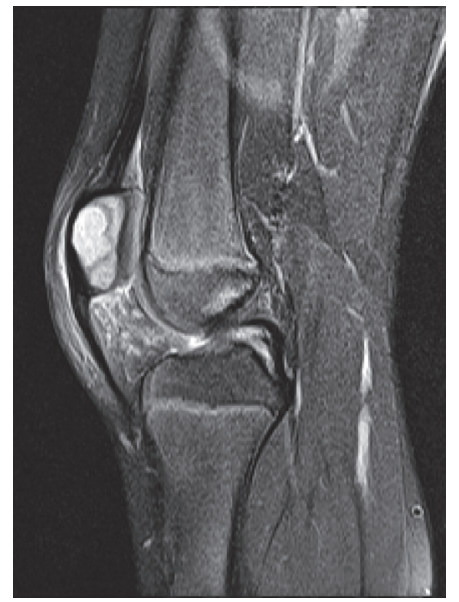

FIGURE 2: MRI showing a sharply limited enhancement of the patella. The radiologist suspected a chondroblastoma.

sedimentation rate was $61 \mathrm{~mm} / \mathrm{h}$ (normal range $1-10 \mathrm{~mm} / \mathrm{h}$ ), and the C-reactive protein (CRP) was $9.3 \mathrm{mg} / \mathrm{dl}$ (normal range $0.0-1.1 \mathrm{mg} / \mathrm{dl})$. A CT scan revealed a $2.0 \times 1.1 \times 1.0 \mathrm{~cm}$ osteolytic lesion of the patella associated with a destruction of the ventral corticalis (Figure 3).

The girl was now referred to our Pediatric Orthopedic Centre. The girl presented with slight swelling of the prepatellar soft-tissue and local hyperthermia of her left knee without joint effusion or fever. Biopsy and a swap were performed in general anaesthesia using a ventral approach. Histological examination showed signs of acute osteomyelitis. Therefore, debridement and curettage were indicated. Intraoperatively, the bone defect measured $1 \times 1.5 \times 1 \mathrm{~cm}$. A gentamicin chain (Septopal Minikette, Pharmalog Pharma Logistik GmbH, Boennen, Germany) was placed into the defect and removed after seven days. The histological examination of the curettage verified acute osteomyelitis and Staphylococcus aureus was grown from the culture. Additionally, the patient was treated with intravenous cefuroxime $(100 \mathrm{mg} / \mathrm{kg}$ bodyweight in three doses) and fosfomycin (190 mg/kg bodyweight in three doses) for 12 days followed by 16 days of oral antibiotics using amoxicillin and clavulanic acid $(37 \mathrm{mg} / \mathrm{kg}$ bodyweight in three doses). The left leg was immobilized in a long leg cast. Following a hospital stay of 17 days the girl was discharged free of pain and the immobilization was continued for another two weeks.

Two weeks later the patient was seen in the outpatient department. She was free of pain and the clinical examination of the knee was unremarkable. CRP and the white blood cell count were within normal ranges. The cast was removed and the patient was sent home without restrictions for daily life activities.

At the latest follow-up examination after 17 months the girl was symptom-free with full range of knee joint motion. Radiographs showed a nearly full osseous recovery of the patella (Figure 4).

\section{Discussion}

The incidence of osteomyelitis during growth is described with 2.9 per 100,000 children with declining values in industrialized countries [16]. A peak of incidence has been found in children aged between 5 and 15 years $[1,7,9,10$, $13,17,18]$. In this age group, osteomyelitis is most commonly caused by haematogenous spread of an infection with an occult source $[2,9,19]$. While most cases of osteomyelitis are diagnosed in the long bones, involvement of the patella is rarely diagnosed $[2,19]$. The patella possesses a rich anastomotic arterial network consisting of contributions from the superior and inferior geniculates and the anterior tibial recurrent arteries [18]. The high number of immune cells in such an extensive vascular network might explain the rare occurrence of haematogenous osteomyelitis of the patella $[1,9,18]$. Additionally, the patella does not have a physeal plate and the associated sluggish hemodynamics have also been considered to explain the rarity of this disease in this location $[1,9]$. 

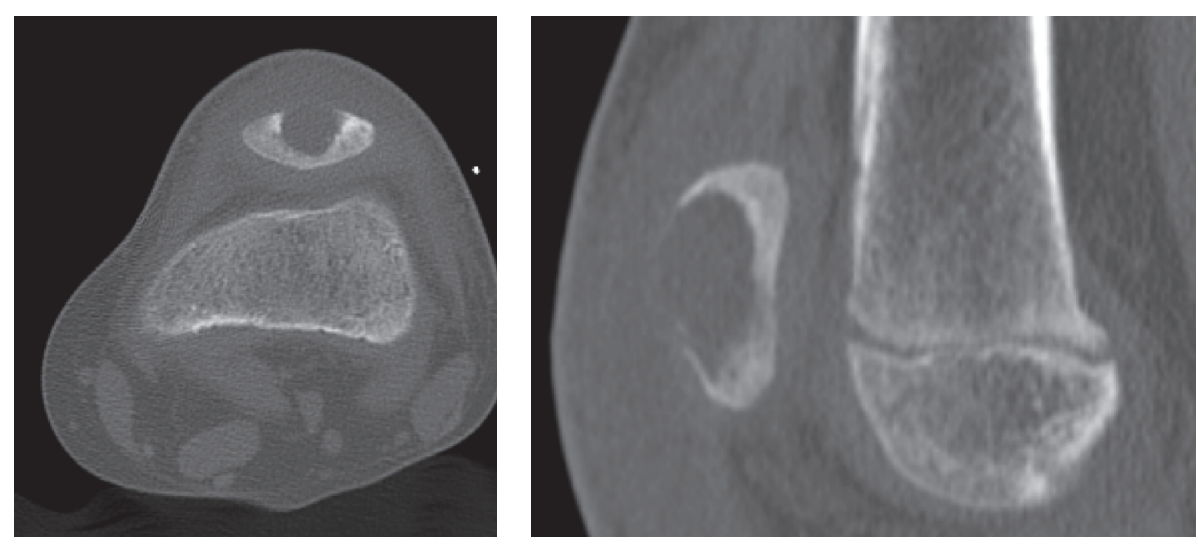

FIGURE 3: CT of the left knee clearly showing an osteolytic process with sharp borders.
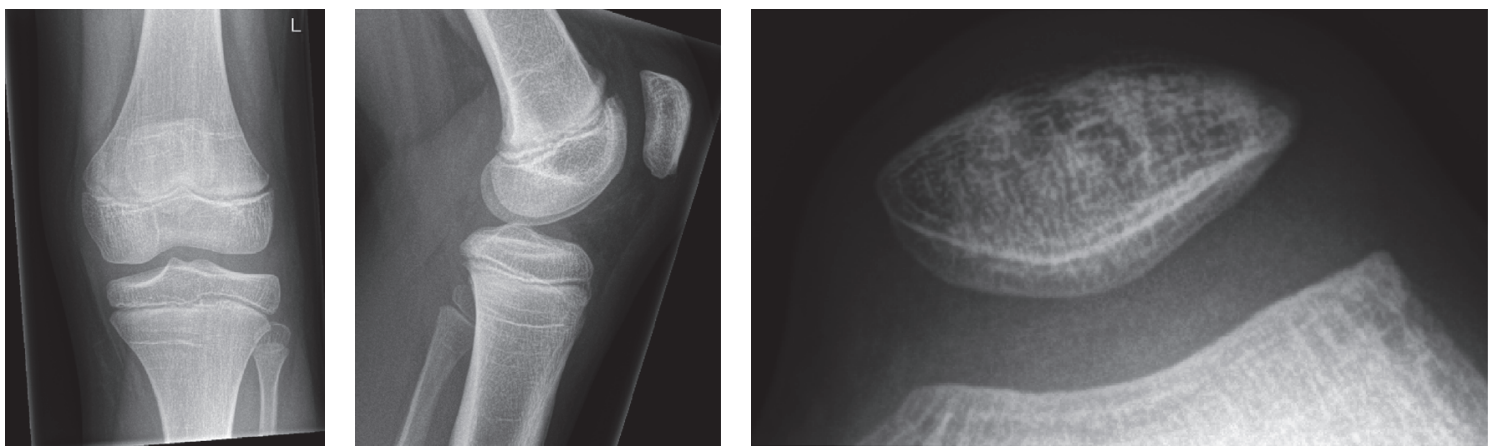

FIGURE 4: Follow-up radiographs 17 months after surgery revealing an almost normally appearing patella.

The correct diagnosis of osteomyelitis of the patella is often delayed with initially suspected diagnoses including septic arthritis, synovitis, septic bursitis, and peripatellar cellulitis [7]. Other differential diagnoses of patellar lesions include Sinding-Larsen-Johansson disease, bipartite patella, and a variety of rare tumors such as chondroblastomas, giant cell tumors, osteoid osteomas, and aneurysmal bone cysts but also malignant tumors [20]. In the patient described in the present case report chondroblastoma was primarily suspected on both conventional radiographs and MRI. The underlying reasons for the late diagnosis are the rarity and the variable presentation of the disease. In children with osteomyelitis of the patella, knee pain can range from mild to severe eventually associated with limping and/or restriction of joint motion. Moreover, leucocyte count and plane radiographs can be inconspicuous early in the course of the disease $[1,9,13,17]$. Due to these variabilities, bone scintigraphy, CT, and gadolinium enhanced MRI have been recommended as diagnostic modalities $[9,17]$. Even though growth disturbances due to patellar osteomyelitis are uncommon, spreading to other parts of the skeleton or local intrusion into the knee joint with subsequent purulent arthritis represents a feared complication. Therefore, prompt diagnosis and therapy are still demanded. To ensure this, a high index of suspicion, close clinical monitoring, and early advanced imaging are highly recommended in children with persistent peripatellar swelling, pain, hyperthermia, and particularly fever and/or elevated inflammatory parameters.

Our review of the literature revealed that reports describing pediatric osteomyelitis of the patella are confined to case reports or very small case series. In total we were able to analyze 14 reports including 27 children. Detailed information about treatment and cultures of these patients is presented in Table 1. Even though there are cases with negative bacterial cultures, Staphylococcus aureus, like in the present case report, seems to represent the most commonly found infecting organism $[2,19]$. Treatment with antibiotics is undisputed and may even be enough for uncomplicated cases of patellar osteomyelitis [19]. However, the duration and the preferred routes of administration, that is, oral or parenteral, greatly vary between the different reports (see Table 1). In accordance with Dartnell et al., surgical treatment consisting of debridement and curettage represents the recommended treatment for absent improvement despite antibiotic treatment and complicated patients like the girl described in the present report $[1,19]$. In patients without joint involvement, arthrotomy does not seem to be necessary. However, no consensus exists regarding the use of antibiotic chains. Advantages of the application of antibiotic chains include the possibility of delivering high local doses of antibiotics without the nephrotoxic and ototoxic side effects [21]. The great disadvantage, however, is the necessity of a second operation 


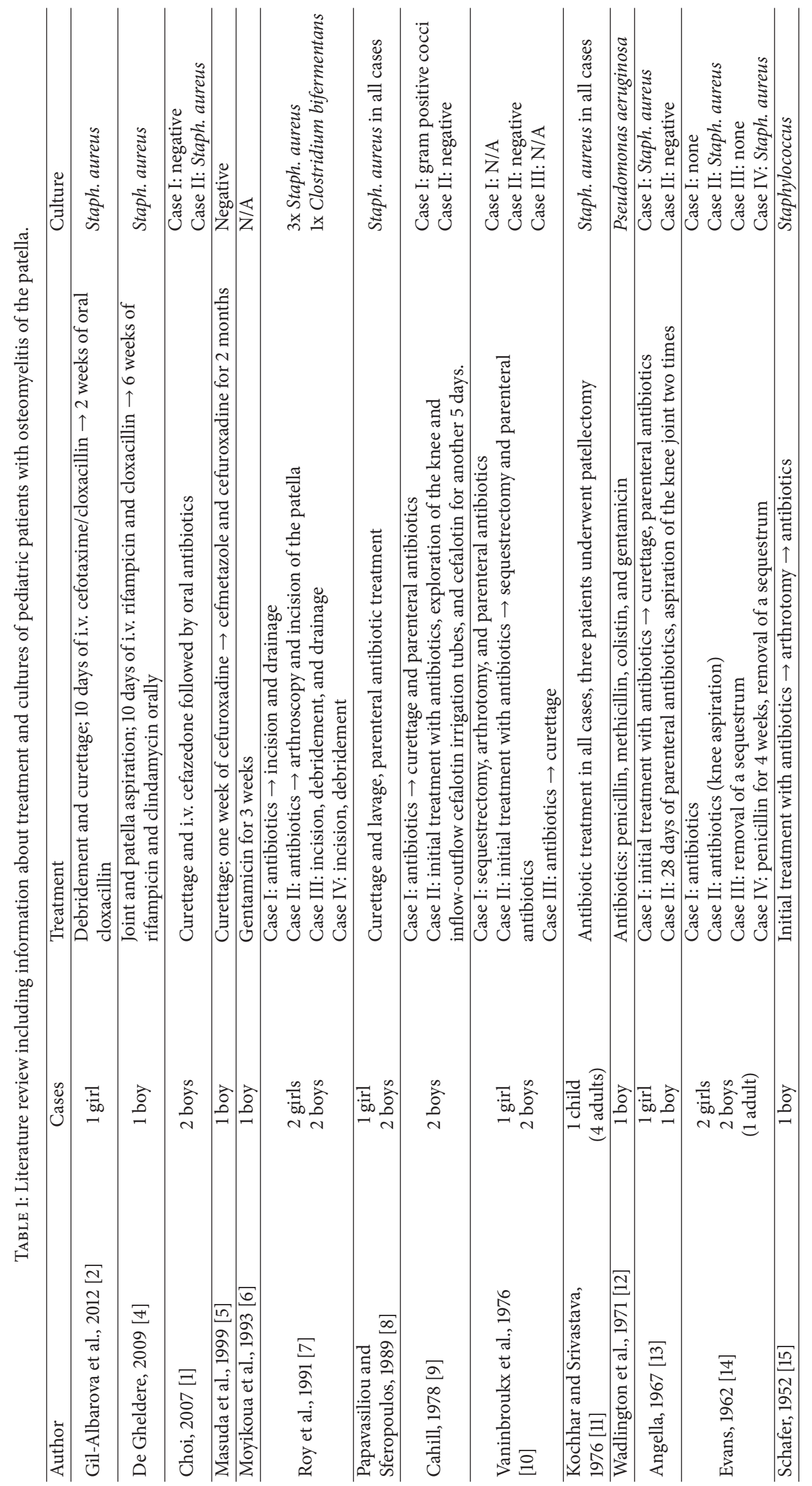


for chain removal. Nevertheless, our treatment regimen consisting of parenteral and subsequent oral antibiotic treatment combined with local gentamicin therapy via a chain led to a favourable outcome despite late diagnosis.

In conclusion, correct diagnosis of pediatric patellar osteomyelitis is frequently delayed. In order to ensure prompt and correct diagnosis, an exact clinical examination, laboratory tests, plain radiographs, and in equivocal cases MRI are indispensable.

\section{Conflicts of Interest}

The authors declare that there are no conflicts of interest regarding the publication of this article.

\section{References}

[1] H.-R. Choi, "Patellar osteomyelitis presenting as prepatellar bursitis," Knee, vol. 14, no. 4, pp. 333-335, 2007.

[2] J. Gil-Albarova, V. E. Gómez-Palacio, and A. Herrera, "Hematogenous osteomyelitis of the patella," Journal of Pediatric Orthopaedics B, vol. 21, no. 5, pp. 411-414, 2012.

[3] Thirion, "Successful extirpation of the right patella for carieas," Lancet, vol. 2, article 399, 1829.

[4] A. De Gheldere, "Haematogenous osteomyelitis of the patella in a child," Acta Orthopaedica Belgica, vol. 75, no. 4, pp. 554-556, 2009.

[5] S. Masuda, T. Shinozaki, H. Watanabe, K. Takagishi, and T. Fukuda, "Acute osteomyelitis of the patella in a child," Japanese Journal of Rheumatology, vol. 9, no. 2, pp. 189-194, 1999.

[6] A. Moyikoua, J. C. Bouity-Buang, Ngatse-Oko, and B. PenaPitra, "Acute osteomyelitis of the patella," Acta Orthopaedica Belgica, vol. 59, no. 1, pp. 98-99, 1993.

[7] D. R. Roy, W. B. Greene, and J. G. Gamble, "Osteomyelitis of the patella in children," Journal of Pediatric Orthopaedics, vol. 11, no. 3, pp. 364-366, 1991.

[8] V. A. Papavasiliou and N. K. Sferopoulos, "Acute hematogenous osteomyelitis of the patella. Apropos of 3 cases," Revue de Chirurgie Orthopédique et Réparatrice de l'Appareil Moteur, vol. 75, pp. 130-132, 1989.

[9] B. R. Cahill, "Nontraumatic osteomyelitis of the patella," Clinical Orthopaedics and Related Research, pp. 177-179, 1978.

[10] J. Vaninbroukx, M. Martens, M. Verhelst, and J. C. Mulier, "Haematogenous osteomyelitis of the patella: report of three cases," Acta Orthopaedica, vol. 47, no. 5, pp. 566-569, 1976.

[11] V. L. Kochhar and K. K. Srivastava, "Unusual lesions of the patella," Int Surg, vol. 61, pp. 37-39, 1976.

[12] W. B. Wadlington, H. Hatcher, and D. J. Turner, "Osteomyelitis of the patella: gentamicin therapy associated with encephalopathy," Clinical Pediatrics, vol. 10, no. 10, pp. 577-580, 1971.

[13] J. J. Angella, "Osteomyelitis of the patella," American Journal of Diseases of Children, vol. 113, no. 5, pp. 590-593, 1967.

[14] D. K. Evans, "Osteomyelitis of the patella," J Bone Joint Surg, vol. 44B, pp. 319-323, 1962.

[15] R. Schafer, "Osteomyelitis of patella," Zentralbl Chir, vol. 77, pp. 425-427, 1952.

[16] M. J. G. Blyth, R. Kincaid, M. A. C. Craigen, and G. C. Bennet, "The changing epidemiology of acute and subacute haematogenous osteomyelitis in children," Journal of Bone and Joint Surgery-Series B, vol. 83, no. 1, pp. 99-102, 2001.
[17] R. K. Kankate and T. P. Selvan, "Primary haematogenous osteomyelitis of the patella: a rare cause for anterior knee pain in an adult," Postgraduate Medical Journal, vol. 76, no. 901, pp. 707709, 2000.

[18] D. R. Roy, "Osteomyelitis of the patella," Clinical Orthopaedics and Related Research, no. 389, pp. 30-34, 2001.

[19] J. Dartnell, M. Ramachandran, and M. Katchburian, "Haematogenous acute and subacute paediatric osteomyelitis," Journal of Bone and Joint Surgery-Series B, vol. 94, no. 5, pp. 584595, 2012

[20] M. Song, Z. Zhang, Y. Wu, K. Ma, and M. Lu, "Primary tumors of the patella," World Journal of Surgical Oncology, vol. 13, no. 1, 2015.

[21] K. Klemm, "The use of antibiotic-containing bead chains in the treatment of chronic bone infections," Clinical Microbiology and Infection, vol. 7, no. 1, pp. 28-31, 2001. 


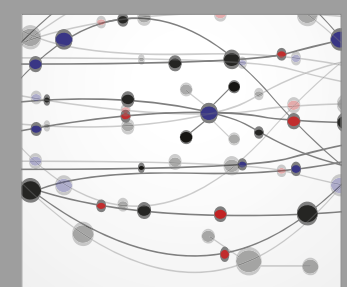

The Scientific World Journal
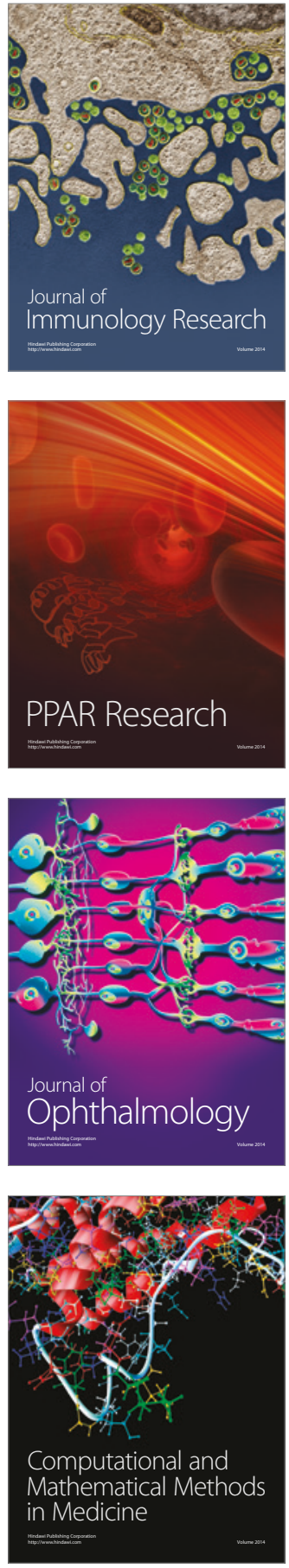

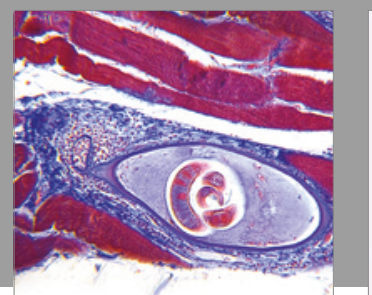

Gastroenterology Research and Practice
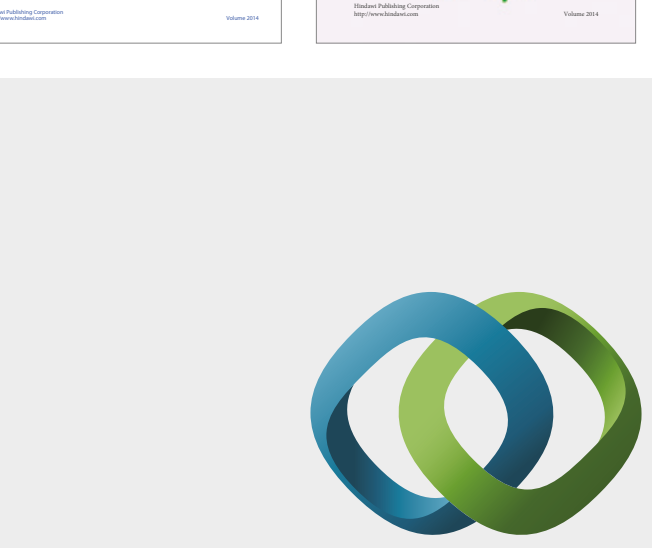

\section{Hindawi}

Submit your manuscripts at

https://www.hindawi.com
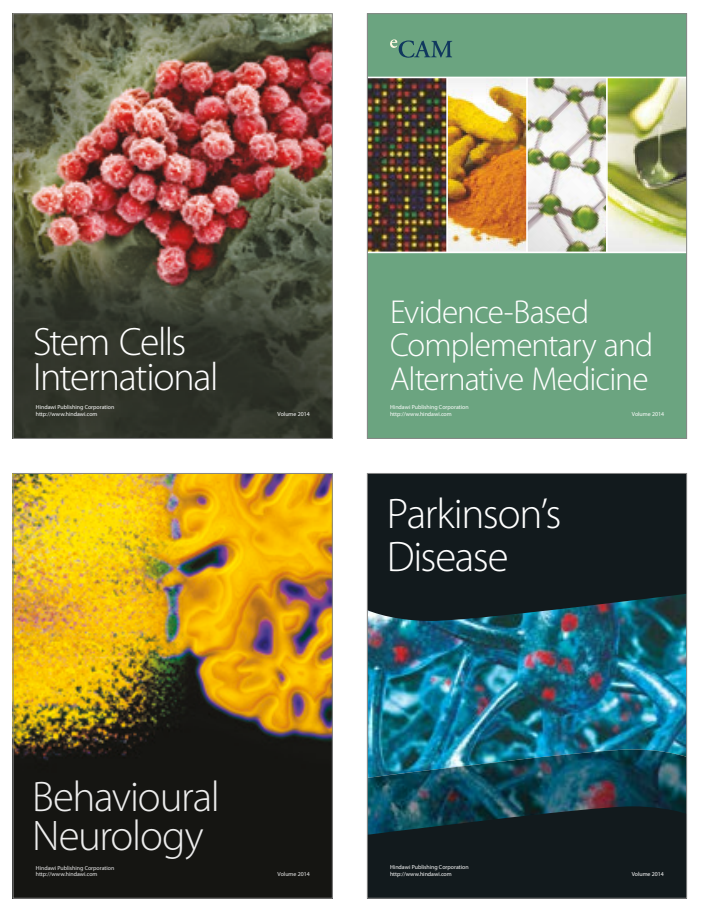
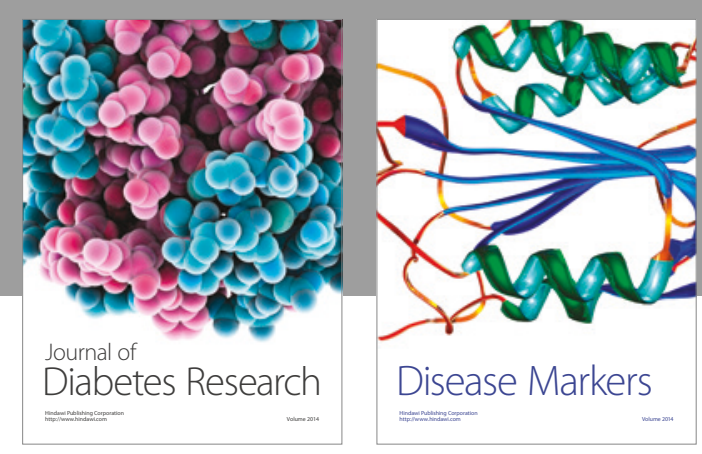

Disease Markers
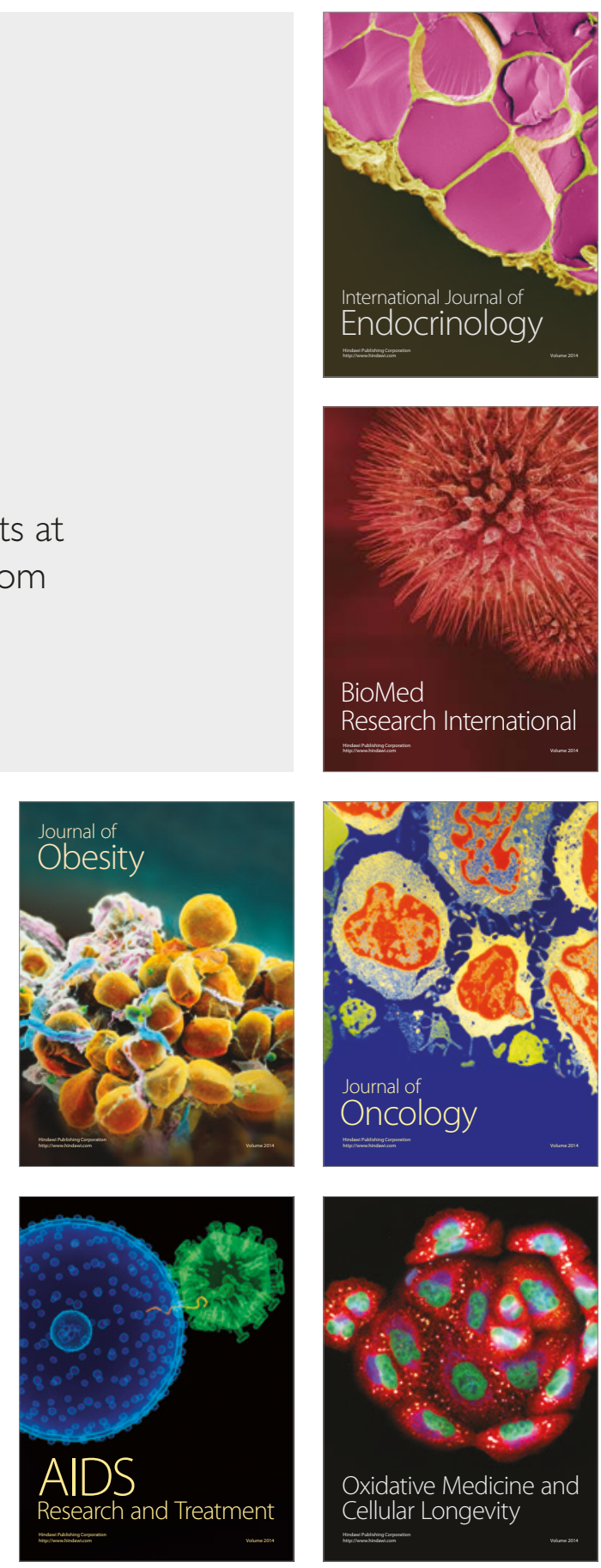